\title{
Intractable/ Persistent Hiccups Due to Acute Subdural Haemorrhage as a Result of Prolonged Inr in a 55 Year Old Man with Rheumatic Mitral Valvular Heart Disease and Atrial Fibrillation: A Case Report
}

\author{
Khin Phyu Pyar ${ }^{1 *}$, Sai Aik Hla ${ }^{2}$, Win Myint ${ }^{3}$, Win Kyaw Shwe ${ }^{4}$, Soe Win Hlaing ${ }^{5}$ Zaw Min Tun ${ }^{6}$, Han Lin Aung ${ }^{7}$, Phyo Htet Oo ${ }^{7}$, Kyaw \\ Zayar Lin ${ }^{7}$, Moe Tun Zaw ${ }^{7}$, Kyaw Zay Ya ${ }^{8}$, Myo Thant Kyaw', Nay Myo Aung', Than Naing Lin', Soe Moe Htun', \& Zay Phyo Aung9 \\ ${ }^{1}$ Professor and Head/Senior Consultant Physician, Department of Medicine/ Department of Nephrology, Defence Services Medical Academy, No.(1) \\ Defence Services General Hospital, Myanmar. \\ ${ }^{2}$ Senior Consultant Physician, Department of Medicine, No. (2) Defence Services General Hospital, Myanmar. \\ ${ }^{3}$ Associate Professor and Consultant Neuro Physician, Department of Medicine, No. (1) Defence Services General Hospital, Myanmar. \\ ${ }^{4}$ Senior Consultant Physician/Nephrologist, Department of Medicine, No. (2) Defence Services General Hospital, Myanmar. \\ ${ }^{5}$ Senior Consultant Physician, Department of Medicine, No. (1) Defence Services General Hospital, Myanmar. \\ ${ }^{6}$ Dip.Med.Ed, Lecturer, Department of Chest Medicine, Defence Services Medical Academy, Myanmar. \\ ${ }^{7}$ Assistant Lecturer, Department of Medicine, Defence Services Medical Academy, Myanmar. \\ ${ }^{8}$ Assistant Lecturer/Haematologist, Department of Medicine, Defence Services Medical Academy, Myanmar. \\ ${ }^{9}$ Assistant Lecturer, Department of Medicine, Defence Services Medical Academy, Myanmar.
}

*Corresponding Author: Khin Phyu Pyar, Professor and Head/Senior Consultant Physician, Department of Medicine/ Department of Nephrology, Defence Services Medical Academy, No. (1) Defence Services General Hospital, Myanmar.

Received Date: 10 October, 2021 | Accepted Date: 28 December, 2021 | Published Date: 10 January, 2022

Citation: Khin P Pyar, Sai A Hla, W Myint, Win K Shwe, Soe W Hlaing, et al. (2022). Intractable/ Persistent Hiccups Due to Acute Subdural Haemorrhage as a Result of Prolonged Inr in a 55 Year Old Man with Rheumatic Mitral Valvular Heart Disease and Atrial Fibrillation: A Case Report. Biomedical Research and Clinical Reviews. 6(1); DOI: 10.31579/2692-9406/089

Copyright: () 2022 Khin Phyu Pyar, This is an open-access article distributed under the terms of the Creative Commons Attribution License, which permits unrestricted use, distribution, and reproduction in any medium, provided the original author and source are credited.

\begin{abstract}
:
A 58 year old man was brought to hospital in state of deep coma following severe headache for one day. His GCS was 3/15 and had flaccid all 4 limbs with equivocal planter response on both sides on arrival. NECT head showed acute subdural haemorrhage with surrounding cerebral oedema, mid-line shift and corning of brain stem. After giving mannitol and dexamethasone, four hours later, he became fully conscious and orientated; his motor power returned to normal. He was on warfarin $3 \mathrm{mg}$ daily for rheumatic mitral valvular heart disease with atrial fibrillation and his INR on arrival was 3.5. He had intractable hiccups once he regained consciousness. Both pharmacological and non-pharmacological measures were tried for distressing hiccups; there was no therapeutic success. His hiccups disappeared completely only after removing the haematoma by burr hole surgery.

Key words: coma; headache; acute subdural haemorrhage; rheumatic mitral valve disease; atrial fibrillation; warfarin; INR; intractable hiccups; burr hole surgery
\end{abstract}




\section{Introduction}

Hiccups is caused by involuntary contraction of diaphragm followed by the rapid closure of vocal cords. Though most of the people experience hiccups from time to time; they usually resolve without treatment within few minutes. If a bout lasts more than 48 hours; it is generally considered as persistent hiccup. And if it is longer than one month; it becomes intractable one. Intractable hiccups may result from many etiologies: structural or functional disturbances at medulla; afferent nerves or efferent nerves to the respiratory muscles; metabolic disorder; endocrine disorder; drugs; general anesthesia and emotional problems. Thus; several reports mentioned structural lesion near medulla producing persistent hiccups; aneurysm; haemorrhage or benign tumor (Musumeci et al.; 2000) (Nagayama et al.; 2004) (Li et al.; 2000) (Hatae et al.; 2014) (Gambhir et al.; 2010) (Chon et al.; 2020) (Al Deeb et al.; 1991). However; one report mentioned supratentorial lesion- chronic subdural haematoma; far away from medulla; may give rise to hiccups (Takemoto et al.; 2015).

One of the fatal side effects of warfarin is haemorrhage in the brain: intracerebral haemorrhage; cerebellar haemorrhage and subdural haemorrhage; and the mortality rate is very high. Several reports mentioned both pharmacological treatment (Lee et al.; 2010) (McGrane et al.; 2015); surgery (Tariq et al.; 2021) and non-pharmacological treatment for intractable hiccup. Among them; one patient with chronic subdural haematoma; intractable hiccups disappeared completely after surgical removal of haematoma (Takemoto et al.; 2015).

\section{Case presentation}

A 58 year old gentleman had severe headache for one day and was found unconscious in bed when his colleagues tried to wake him up in the morning. He vomited twice on the way to hospital. He was known to have rheumatic mitral valvular heart disease (mitral stenosis and mitral regurgitation) with atrial fibrillation and he was on warfarin $3 \mathrm{mg}$ OD; penicillin V $500 \mathrm{mg} \mathrm{BD}$; digoxin $0.5 \mathrm{mg}$ OD; telmisartan $20 \mathrm{mg}$ OD and frusemide $20 \mathrm{mg}$ OD $\mathrm{cm}$ alternate day.His previous INR were varied between 2 to 3; but it was never above 3. However; he did not check INR for 3 weeks as he was busy. There was no history of fever or head injury.

On arrival; his Glasgow Coma Scale was 3/15 and had flaccid all 4 limbs with equivocal planter response on both sides. He was afebrile; his $\mathrm{SaO} 2$ was $95 \%$ on air; his blood pressure was $140 / 90 \mathrm{mmHg}$; and his pulse rate $62 /$ min with irregularly irregular in character. He had both pansystolic murmur and mid diastolic murmur in mitral area and there was no features of heart failure or infective endocarditis.

NECT head showed acute subdural haemorrhage with surrounding cerebral oedema; mid-line shift and corning of brain stem. (Figure 1) Four hours later; after giving mannitol and dexamethasone; he became fully conscious and orientated. Motor power returned to normal. His INR on arrival was 3.5. Therefore; the rapid reversal of anticoagulation was done; warfarin was withheld; and parenteral vitamin $\mathrm{K} 10 \mathrm{mg}$ was given.The coagulation status became normal after infusion of fresh frozen plasma and $10 \mathrm{mg}$ vitamin $\mathrm{K}$ replacement.Haemostatic agent (tranxenamic acid) was given too. Twenty-four hour after rapid reversal of anti-coagulation; the patient did not suffer headache and clinical signs of raised intracranial pressure (blood pressure and heart rate) settled down. He had intractable hiccups once he regained consciousness.

Blood for complete picture (Haemoglobin $12.5 \mathrm{gm} \%$; Total WBC $7.0 \times 10^{9} / \mathrm{L}$; neutrophil $75 \%$; lymphocyte $22 \%$; monocyte $3 \%$; plateleta $\left.350 \times 10^{9} / \mathrm{L}\right)$; blood urea and electrolytes; random blood sugar; blood cholesterol and liver function tests were normal. Blood culture was sterile. ECG revealed absent "p" wave; varying R-R interval with right ventricular hypertrophy. Chest radiograph showed mild cardiomegaly. In echocardiogram; there was no clot in left atrium and mitral valve orifice was $4 \mathrm{~mm}^{2}$ with some calcification.

We had to try for the best for both; prevention of clot in left atrium and cessation of further bleed in the brain. He received $20 \mathrm{mg}$ enoxaparin subcutaneously 10 hours after the operation; and afterward at least $40 \mathrm{mg}$ enoxaparin was administered daily for thromboembolism prophylaxis.

Hiccups was disturbing his eating and drinking: even he could not sleep. Various pharmacological (diazepan; metoclopramide; haloperidol) and non-pharmacological measures (drinking ice water; application of ice pad; breath holding; distraction of attention; Valsalva maneuver) were tried but to no evil.

He was completely free of hiccups only after evacuation of blood by burr hole. CT head after evacuation showed reduction in volume of haematoma and resolution of cerebral oedema. And there was no mid-line shift. (Figure 2) The post-operative period was uneventful.

We planned for mitral valve replacement for his underlying mitral stenosis and mitral regurgitation and advised to continue penicillin for prevention of further rheumatic activity. We remind the patient not to miss "the warfarin clinic" again.

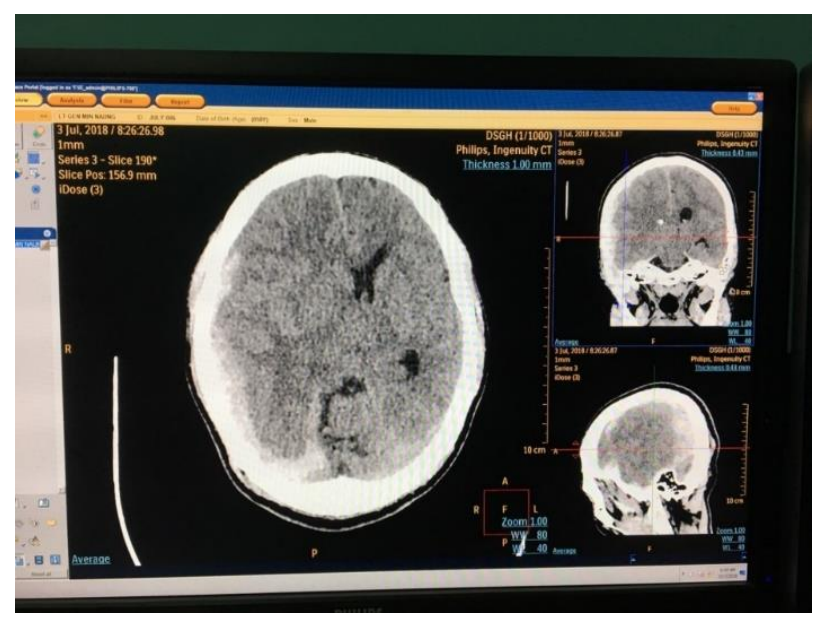

Figure 1: CT head on arrival to $A \& E$ 

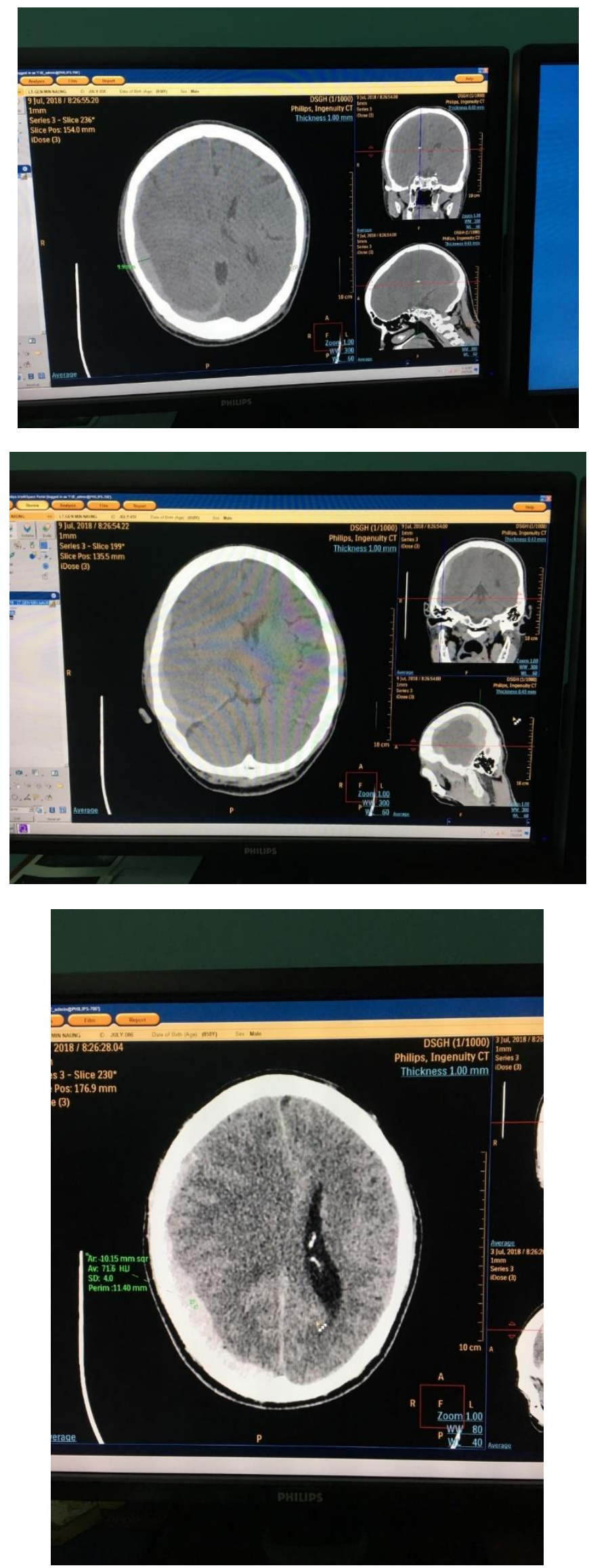

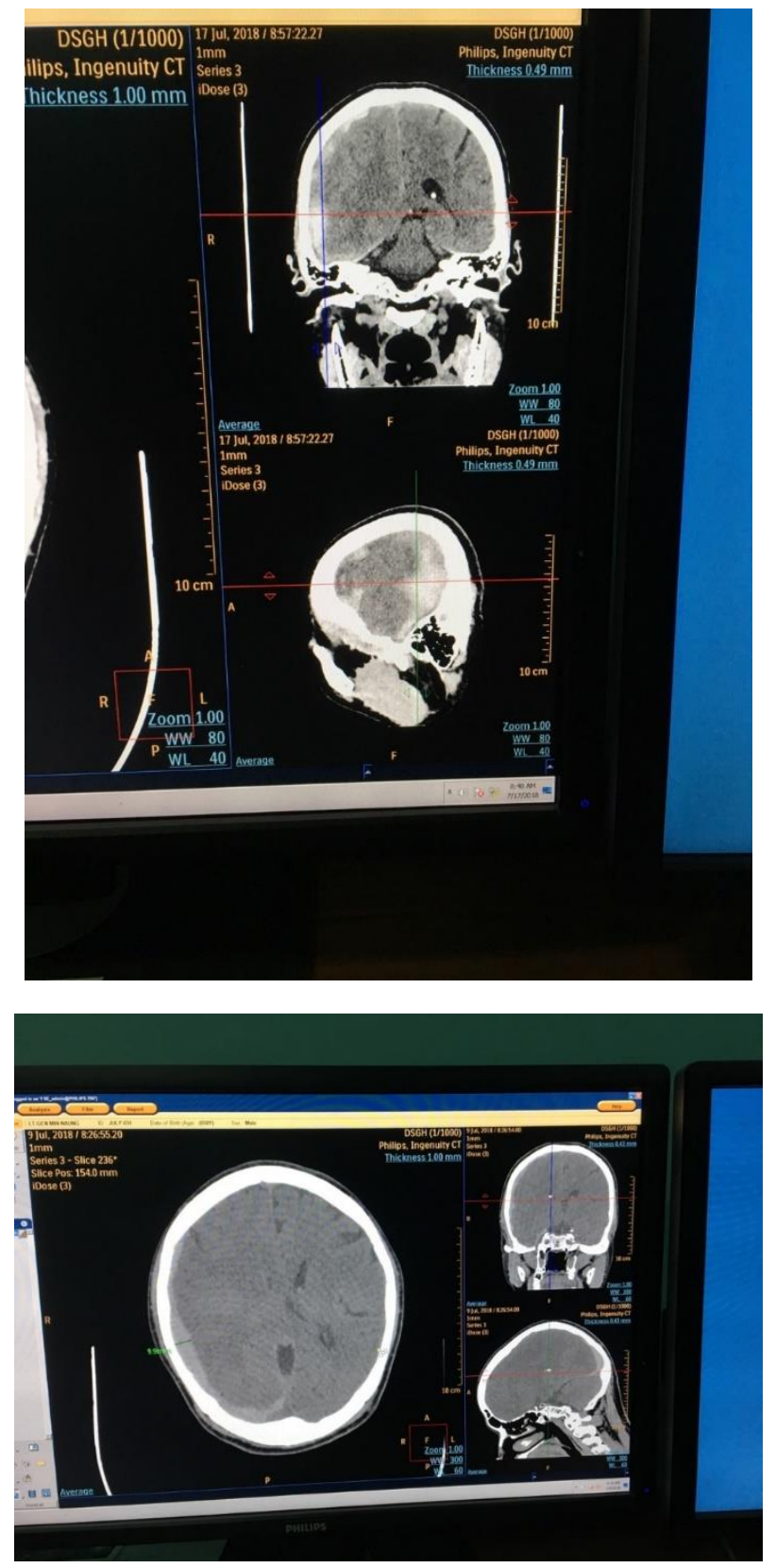

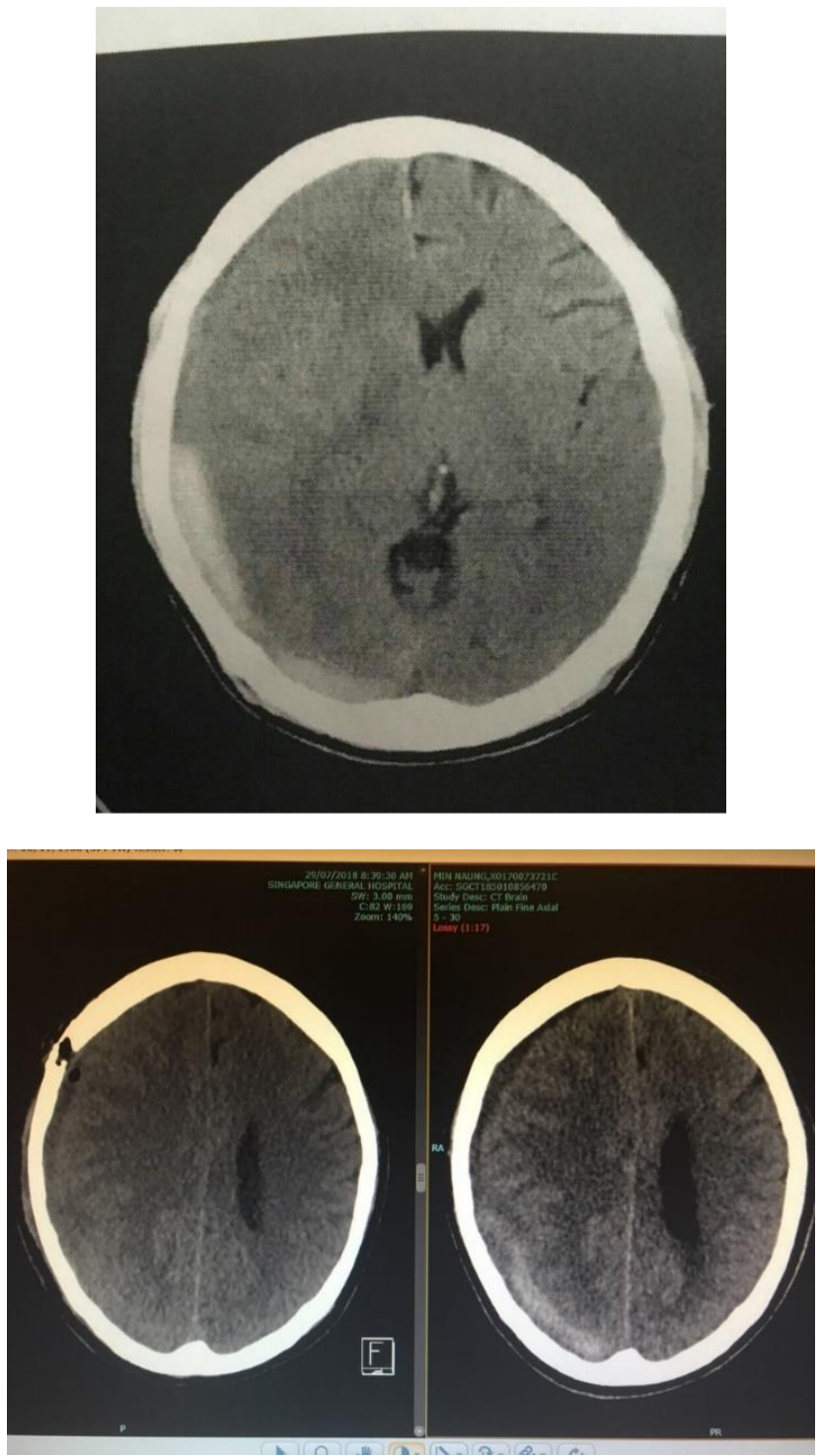

Figure 2: CT head after burr hole evacuation

\section{Discussion}

Hiccups was well known for one of the features of lateral medullary syndrome and there were several case reports on it (Sampath et al.; 2014). And the structural abnormalities near the medulla leading to intractable hiccups was also reported in some cases having vascular or tumor lesions close to medulla (Musumeci et al.; 2000)(Nagayama et al.; 2004)(Li et al.; 2000)(Hatae et al.; 2014)(Gambhir et al.; 2010)(Chon et al.; 2020)(Al Deeb et al.; 1991).

However; hiccups was seen as one of presenting symptoms in a case with chronic subdural haematoma and the patient was free of symptom after evacuation of blood (Takemoto et al.; 2015).Thus; it is possible that supratentorial areas involve in the either stimulation or suppression of the hiccups centers though these areas are far away from medulla. None of the report explained about distressing hiccups in acute subdural haematoma. Thus; it was the main reason for presenting this case.

Moreover; the role of warfarin was essential in this case to prevent thrombus formation in left atrium though it was not seen in transthoracic echocardiogram. On the other hand; limitation of further expansion of acute subdural haemorrhage was paramount importance for neurological sequelae. Thus; appropriate timing for evacuation of haematoma with minimum bleeding risks was also the art of medicine. It was another reason to share our knowledge. Lin et al (2017) pointed out that the mortality rate of patients with acute subdural haematoma already on long warfarin therapy was nearly double than those who were not on warfarin. Thus; this case was one of the rare cases.

Furthermore; after surgery; monitoring for prevention of rebleeding was very delicate as many case reports mentioned high rebleeding risks (Gonugunta \& Buxton; 2001). Restarting warfarin therapy was done 2 weeks after burr hole surgery. He has no evidence of rebleed till now- 18 months after surgery.

Health education - "importance of warfarin clinic" was extremely crucial as missing one appointment led to torrential fatal event.

\section{Conclusion}

Both prevention of thromboembolism in high risks patient- atrial fibrillation in mitral valve disease with anti-coagulation and retardation 
of extension of acute subdural haemorrhage are difficult therapeutic dilemma in this patient. Initially; rapid reversal of anti-coagulation saved the life. Timing and preparation for evacuation of haematoma is critical. After evacuation; prevention of rebleeding is also important. However; the main concerned from patient point ie the most distressing symptom was persistent hiccups which disappeared completely after surgery.

\section{Acknowledgements}

The authors would like to thank the patient for giving consent to this article. Also; to all doctors and nursing team for making great efforts in caring him. The authors acknowledged the following team; cardiac medical team - Dr Win Thaw; neuro surgical team - Dr Thant Lwynn San; intensive care team - Prof Yu Aye Latt; Dr Chan Myae \& Dr Nay Myo Lwin; Laboratory team - Professor Tin Moe Mya; radiological teamProfessor Kyaw Za Ya; administrative support - Prof Soe Win; Prof Soe Hlaing and Prof Myint Zaw.

\section{Declaration of conflict of interest}

The authors declared no potential conflicts of interests with respect to authorship and publication of this article.

\section{Ethical approval}

Our institution does not require ethical approval for reporting cases.

\section{Funding}

The authors received no financial support for publication of this article.

\section{Informed consent}

The informed consent for publication in this article was obtained from patient.

\section{References}

1. Al Deeb S. M, Sharif H, Al Moutaery K \& Biary N. (1991). Intractable hiccup induced by brainstem lesion. Journal of the Neurological Sciences. 103(2):144-150.

2. Chon J, Yoo S. D, \& Lee S. A. (2020). Hiccups triggered by bladder filling after bilateral pontine hemorrhage: A case report. Medicine. 99(14):19338-19338.

3. Gambhir S, Singh A, Maindiratta B, Jaeger M, Darwish B \& Sheridan M. (2010). Giant PICA aneurysm presenting as intractable hiccups. Journal of Clinical Neuroscience; 17(7); 945 946.

4. Gonugunta V \& Buxton N. (2001). Warfarin and chronic subdural haematomas. British Journal of Neurosurgery, 15(6):514-517.

5. Hatae R, Miyazono M, Kohri R, Maeda K \& Naito S. (2014). Trochlear nerve schwannoma with intratumoral hemorrhage presenting with persistent hiccups: A case report. Journal of Neurological Surgery Reports. 75(1):183-188.

6. Kumar A. \& Dromerick A. W. (1998). Intractable hiccups during stroke rehabilitation. Archives of Physical Medicine and Rehabilitation. 79(6):697-699.

7. Lee J. H, Kim T. Y, Lee H. W, Choi Y. S, Moon S. Y \& Cheong Y. K. (2010). Treatment of intractable hiccups with an oral agent monotherapy of baclofen a case report. The Korean Journal of Pain. 23(1):42-45.

8. Li M, Gupta A, Thomas P, \& Richards A. (2000). Basilar artery aneurysm: An unusual cause of intractable hiccups. Hospital Medicine. 61(12):868-869.

9. Lin L M, Paff M, Xu R, Jiang B, Colby G. P \& Coon A. L. (2017). Chronic anticoagulation with warfarin is associated with decreased functional outcome and increased length of stay following craniotomy for acute subdural hematoma. Interdisciplinary Neurosurgery. 8:35-39.

10. McGrane I. R, Shuman M. D, \& McDonald R. W. (2015). Donepezil-Related Intractable Hiccups: A Case Report. Pharmacotherapy: The Journal of Human Pharmacology and Drug Therapy. 35(3):1-5.

11. Musumeci A, Cristofori L \& Bricolo A. (2000). Persistent hiccup as presenting symptom in medulla oblongata cavernoma: A case report and review of the literature. Clinical Neurology and Neurosurgery. 102(1):13-17.

12. Nagayama T, Kaji M, Hirano H, Niiro M \& Kuratsu J. (2004). Intractable hiccups as a presenting symptom of cerebellar hemangioblastoma. Journal of Neurosurgery. 100(6): 1107-1110.

13. Rizzo C, Vitale C \& Montagnini M. (2013). Management of Intractable Hiccups: An Illustrative Case and Review. American Journal of Hospice and Palliative Medicine®. 31(2):220-224.

14. Sampath V, Gowda M. R, Vinay H. R, \& Preethi S. (2014). Persistent hiccups (singultus) as the presenting symptom of lateral medullary syndrome. Indian Journal of Psychological Medicine. 36(3):341-343.

15. Takemoto Y, Hashiguchi A, Moroki K, Tokuda H \& Kuratsu J. (2015). Chronic subdural hematoma with persistent hiccups: A case report. Interdisciplinary Neurosurgery.

16. Tariq K, Das J. M, Monaghan S, Miserocchi A, \& McEvoy A. (2021). A case report of Vagus nerve stimulation for intractable hiccups. International Journal of Surgery Case Reports. 78:219222.

17. Yeon J Y, Kong D S. \& Hong S C. (2012). Safety of early warfarin resumption following burr hole drainage for warfarin-associated subacute or chronic subdural hemorrhage. Journal of Neurotrauma. 29(7): 1334-1341. 